\title{
GENERATORS AND RELATIONS FOR COXETER GROUPS
}

\author{
C. T. BENSON AND L. C. GROVE
}

The original proof of Coxeter's Theorem on generators and relations of reflection groups was topological in nature. Algebraic proofs were given in [1] and [3]. We present here an algebraic proof, shorter than the previous ones, having the advantage that it admits a geometric interpretation closely related to Coxeter's original proof.

Coxeter's Theorem [2, p. 599]. Suppose $V$ is a real Euclidean vector space and $G$ is a finite subgroup of the orthogonal group $O(V)$ generated by reflections. Then $G$ has a presentation

$$
G=\left\langle S_{i} \mid\left(S_{i} S_{j}\right)^{a_{i j}}=1, \quad a_{i i}=1, \quad 1 \leqq i \leqq j \leqq n\right\rangle .
$$

We use the notation of [1]. The length $l(T)$ of an element $T$ of $G$ is the minimal number of factors possible when $T$ is represented as a word in the fundamental reflections $S_{i}$. The words $S_{i_{1}} \cdots S_{i j}$, with $0 \leqq j \leqq k$, are called partial words of the word $S_{i_{1}} \cdots S_{i_{k}}$. The symbol $\left(S_{i} S_{j} \cdots\right)_{m}$ will denote a word in alternating $S_{i}$ 's and $S_{j}$ 's, beginning with $S_{i}$ and having $m$ factors, $m \geqq 0$. Similar remarks apply to $\left(\cdots S_{i} S_{j}\right)_{m}$ and $\left(\cdots S_{i} S_{j} \cdots\right)_{m}$.

Lemma. Suppose $S \in G, i$ and $j$ are fixed, and $l\left(S S_{i}\right)=l\left(S S_{j}\right)=l(S)-1$. Then $l\left(S\left(\cdots S_{i} S_{j} \cdots\right)_{m}\right)=l(S)-m$ if $0 \leqq m \leqq a_{i j}$.

Proof. The conclusion is trivial if $m=0$, so suppose $m \geqq 1$ and that the result holds for $m-1$. If $a_{i}$ and $a_{j}$ are the fundamental roots corresponding to $S_{i}$ and $S_{j}$, then by Lemma 2.2 of [3] we have $S a_{i}$, $S a_{j} \in-\Sigma$. The reflections $S_{i}$ and $S_{j}$ generate a dihedral group of order $2 a_{i j}$, and it is easy to see that $l\left(\left(\cdots S_{i} S_{j}\right)_{m}\right)=m$. It follows that $\left(\cdots S_{j} S_{i}\right)_{m-1} a_{j} \in \Sigma$, again by Lemma 2.2 of [3], and so $\left(\cdots S_{j} S_{i}\right)_{m-1} a_{j}$ $=\alpha a_{i}+\beta a_{j}$, with $\alpha, \beta \geqq 0$. Thus

$$
S\left(\cdots S_{j} S_{i}\right)_{m-1} a_{j}=\alpha S a_{i}+\beta S a_{j} \in-\Sigma,
$$

and

$$
\begin{aligned}
l\left(S\left(\cdots S_{i} S_{j}\right)_{m}\right) & =l\left(S\left(\cdots S_{j} S_{i}\right)_{m-1}\right)-1=l(S)-(m-1)-1 \\
& =l(S)-m .
\end{aligned}
$$

Received by the editors April 21, 1969 and, in revised form, September 24, 1969. 
Proof of the theOREM. We shall show that every relation $W=S_{i_{1}} \cdots S_{i_{k}}=1$ is a consequence of the relations $\left(S_{i} S_{j}\right)^{a_{i j}}=1$. Suppose $u$ is the maximal length of partial words of $W$. Then we may write $W$ as $W_{1} S_{i} S_{j} W_{2}$, where $l\left(W_{1} S_{i}\right)=u$ and every partial word of $W_{1}$ has length less than $u$. Set $a=a_{i j}$, let $W^{\prime}=W_{1}\left(S_{j} S_{i} \cdots\right)_{2 a-2} W_{2}$, and observe that $W$ and $W^{\prime}$ are equal as elements of $G$. With the exception of $W_{1} S_{i}$ it is clear that all partial words of $W$ are equal, as group elements, to partial words of $W^{\prime}$. In place of $W_{1} S_{i}, W^{\prime}$ has the partial words $W_{1} S_{j}, W_{1} S_{j} S_{i}, \cdots, W_{1}\left(S_{j} S_{i} \cdots\right)_{2 a-3}$. Setting $S=W_{1} S_{i}$, and using the fact that $S_{i}^{2}=1$, we see that the latter partial words coincide as group elements with $S\left(S_{i} S_{j} \cdots\right)_{r}, 2 \leqq v \leqq 2 a-2$. Each of these has length less than $l(S)=u$. This is a direct consequence of the lemma if $v \leqq a$, otherwise it is a consequence of the lemma and the fact that $\left(S_{i} S_{j} \cdots\right)_{v}=\left(S_{j} S_{i} \cdots\right)_{2 a-v}$, since then $2 a-v<a$. Replacing $W$ by $W^{\prime}$ we have removed the first partial word of maximal length in $W$. The procedure may be repeated as necessary until we arrive at the relation $1=1$, and the theorem is proved.

For the geometrical interpretation we associate with each word $S_{i_{1}} \cdots S_{i_{k}}$ a path in $V$ (or in a simply connected subset invariant under $G$ ) as in [2, p. 600]. If $F$ is the initial fundamental region, then the length of a word $S_{i_{1}} \cdots S_{i_{k}}$ is the minimal number of fundamental regions crossed by paths from $F$ to $S_{i_{1}} \cdots S_{i_{k}} F$.

The steps of the proof above may be illustrated for the group $G$ of symmetries of the cube in three dimensions. In this case

$$
G=\left\langle S_{1}, S_{2}, S_{3} \mid S_{i}^{2}=\left(S_{1} S_{2}\right)^{2}=\left(S_{1} S_{3}\right)^{3}=\left(S_{2} S_{3}\right)^{4}=1\right\rangle .
$$

As a simple example of a relation in $G$ we have

$$
W=S_{3} S_{1} S_{2} S_{3} S_{1} S_{3} S_{1} S_{3} S_{2} S_{1} S_{2} S_{1} S_{2} S_{3}=1 .
$$

The corresponding closed path is illustrated in the figure, with Roman numerals indicating lengths of partial words. Thus $S_{3} S_{1} S_{2} S_{3} S_{1}$ is the partial word of maximal length, and $l\left(S_{3} S_{1} S_{2} S_{3} S_{1}\right)=u=5$. Applying the relation $\left(S_{1} S_{3}\right)^{3}=1$ we obtain the relation

$$
W^{\prime}=S_{3} S_{1} S_{2} S_{3} S_{3} S_{1} S_{3} S_{1} S_{1} S_{3} S_{2} S_{1} S_{2} S_{1} S_{2} S_{3}=1
$$

and the path with the dotted line. The new word $W^{\prime}$ has two partial words of length 4 , viz. $S_{3} S_{1} S_{2} S_{3}$ and $S_{3} S_{1} S_{2} S_{3} S_{3} S_{1} S_{3} S_{1}$, but no partial words of length 5 or greater. 


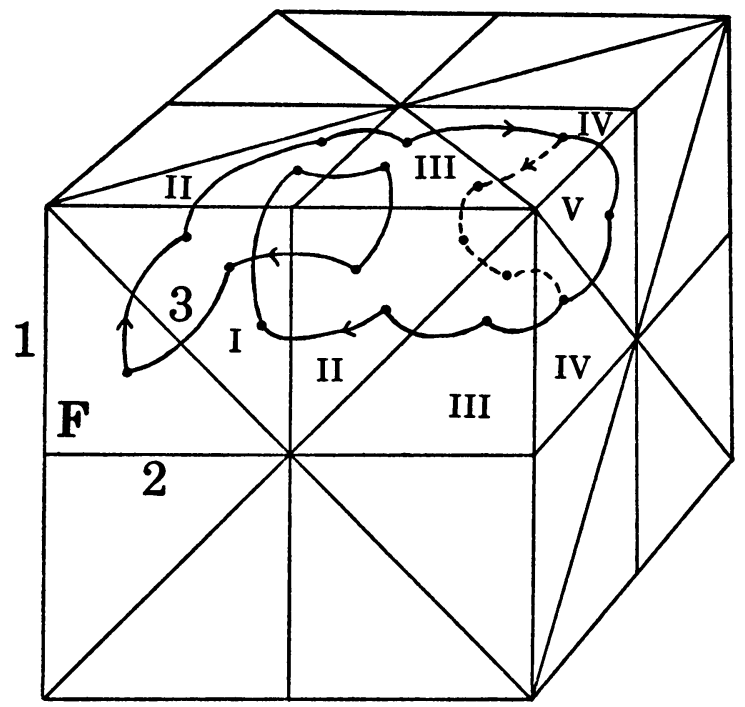

Figure 1

The process continues, giving the further sequence of relations

$$
\begin{aligned}
& S_{3} S_{1} S_{2} S_{1} S_{3} S_{1} S_{1} S_{3} S_{2} S_{1} S_{2} S_{1} S_{2} S_{3}=1, \\
& S_{3} S_{1} S_{2} S_{1} S_{3} S_{3} S_{2} S_{1} S_{2} S_{1} S_{2} S_{3}=1, \\
& \cdot . \cdot . \cdot . \cdot . \cdot . \cdot . \cdot \\
& S_{3} S_{3}=1, \quad \text { and finally } 1=1 .
\end{aligned}
$$

As the example shows, the removal of a partial word of maximal length corresponds to Coxeter's device of shrinking the path through a wall (if $i=j$ ), or past an edge (if $i \neq j$ ), of a fundamental region.

We wish to thank the referee for suggesting a simplification of the proof of the theorem.

\section{REFERENCES}

1. P. Cartier, "Groupes finis engendrés par des symétries," en Séminaire $C$. Chevalley, 1956/58. Classification des groupes de Lie algébriques. Vol. 2, Secrétariat mathématique, Paris, 1958. MR 21 \#5696.

2. H. S. M. Coxeter, Discrete groups generated by reflections, Ann. of Math. (2) 35 (1934), 588-621.

3. N. Iwahori, On the structure of a Hecke ring of a Chevalley group over a finite field, J. Fac. Sci. Univ. Tokyo Sect. I 10 (1964), 215-236. MR 29 \#2307.

THE UNIVERSITY OF OREGON AND

Syracuse University 